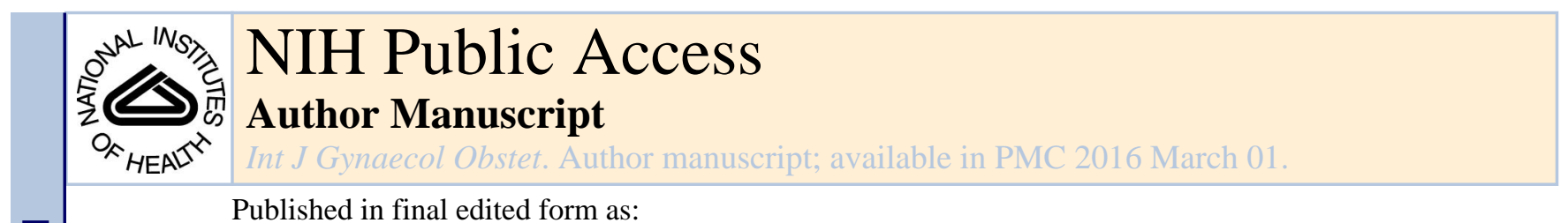

Published in final edited form as:

Int J Gynaecol Obstet. 2015 March ; 128(3): 201-205. doi:10.1016/j.ijgo.2014.09.026.

\title{
Uptake of a women-only, sex-work-specific drop-in center and links with sexual and reproductive health care for sex workers
}

\author{
Rachel Kim ${ }^{a}$, Shira Goldenberg ${ }^{b, c}$, Putu Duff $^{b}$, Paul Nguyen $^{b}$, Kate Gibson ${ }^{d}$, and Kate \\ Shannon ${ }^{\mathrm{b}, \mathrm{c},{ }^{,}}$ \\ aDepartment of Obstetrics and Gynecology, Faculty of Medicine, University of British Columbia, \\ Vancouver, BC, Canada \\ 'Gender and Sexual Health Initiative, BC Centre for Excellence in HIV/AIDS, St. Paul's Hospital, \\ Vancouver, BC, Canada \\ 'Department of Medicine, University of British Columbia, St. Paul's Hospital, Vancouver, BC, \\ Canada \\ dWISH Drop-In Centre Society, Vancouver, BC, Canada
}

\begin{abstract}
Objective-To longitudinally examine female sex workers' (FSWs') uptake of a women-only, sex-work-specific drop-in service and its impact on their access to sexual and reproductive health (SRH) services.

Methods-For the present longitudinal analysis, data were drawn from the AESHA (An Evaluation of Sex Workers' Health Access) study, a community-based, open, prospective cohort of FSWs from Vancouver, BC, Canada. Data obtained between January 2010 and February 2013 were analyzed. Participants are followed up on a semi-annual basis. Multivariable logistic regression using generalized estimating equations was used to identify correlates of service uptake.
\end{abstract}

Results-Of 547 FSWs included in the present analysis, 330 (60.3\%) utilized the services during the 3-year study period. Service use was independently associated with age (adjusted odds ratio [AOR] 1.04; 95\% confidence interval [CI] 1.03-1.06), Aboriginal ancestry (AOR 2.18; 95\% CI 1.61-2.95), injection drug use (AOR 1.67; 95\% CI 1.29-2.17), exchange of sex for drugs (AOR 1.40; 95\%CI 1.15-1.71), and accessing SRH services (AOR 1.65; 95\% CI 1.35-2.02).

\footnotetext{
(C) 2014 International Federation of Gynecology and Obstetrics. Published by Elsevier Ireland Ltd. All rights reserved.

*Corresponding author: Kate Shannon, St. Paul's Hospital, 608-1081 Burrard Street, Vancouver, BC V6Z 1Y6, Canada. Tel.: +1 604 804 9459; fax: +1 604806 9044. gshi@ cfenet.ubc.ca.

Conflict of interest

The authors have no conflicts of interest.

Publisher's Disclaimer: This is a PDF file of an unedited manuscript that has been accepted for publication. As a service to our customers we are providing this early version of the manuscript. The manuscript will undergo copyediting, typesetting, and review of the resulting proof before it is published in its final citable form. Please note that during the production process errors may be discovered which could affect the content, and all legal disclaimers that apply to the journal pertain.
} 
Conclusion-A sex-work-specific drop-in space for marginalized FSWs had high uptake. Women-centered and low-threshold drop-in services can effectively link marginalized women with SRH services.

\section{Keywords}

Health access; Health services; Outreach services; Peer-led; Reproductive health; Sex work; Sexual health; Support services

\section{Introduction}

Sexual and reproductive health (SRH) services-including contraception, prenatal care, family planning, and abortion care-are a crucial component of women's reproductive rights [1]. In many international settings, female sex workers (FSWs) of reproductive age experience high reproductive and sexual health morbidity (e.g. unsafe abortions and genital tract infections) [2] and have suboptimal access to SRH services (e.g. contraception), largely because of social and structural barriers (e.g. stigma, criminalization, and restrictive funding policies) [3-5]. Most research and interventions with FSWs have focused on the prevention, treatment, and care of HIV and other sexually transmitted infections (STIs) [6]. Access and utilization of broader SRH services among FSWs—including positive sexual health resources, contraceptive access, family planning, and pregnancy and prenatal support-have been largely neglected.

Alongside a high burden of HIV and STIs among FSWs relative to the general population of women [2,4,6,7], studies show large SRH disparities, including poor access to pregnancy and parenting services, low rates of cervical cancer screening, a high burden of unwanted pregnancies, and low contraception use [5,8]. However, FSWs face increased risks for reproductive and sexual morbidity because they have multiple sexual partners and encounter gender and economic barriers to male condom use and contraceptive access and use [6,9].

FSWs face significant barriers to SRH access across low-, middle-, and high-income settings $[6,8]$. These barriers include the criminalization of sex work and HIV status non-disclosure, occupational stigma, discrimination by healthcare providers, limited knowledge of services available, reluctance to seek help from healthcare professionals because of mistrust, and social and health inequities (e.g. poverty, mental health issues, illicit drug use, and homelessness) [7-10]. In Vancouver, Canada, FSWs have also been found to face significant challenges while pregnant or caring for a child, including lack of financial support, fear of partner violence, avoidance of services as a result of fear of child apprehension, and stigma [11].

The substantial health disparities and barriers to care experienced by FSWs highlights the need for appropriate, nonjudgmental services and outreach programs, which can promote better SRH access. Most successful strategies to improve FSWs' access to HIV prevention and care have been based on community empowerment models, including health and support services led by FSWs and tailored to their needs $[6,12,13]$. For example, the IMPACT project in Mombasa, Kenya, included a peer-mediated intervention to prevent HIV and STIs, which increased consistent condom use and access to SRH services [14]. Other 
effective strategies have been implemented in low- and middle-income countries (LMICs), such as India [15], Brazil [16], and Mozambique [17]. Although these programs have been shown to successfully link FSWs with health and support services [6], data from highincome settings such as Canada remain scarce.

In Vancouver, BC, Canada, the Women's Information Safe Haven (WISH) Drop-In Centre Society (hereafter, WISH) is a service and support organization for street-involved women in sex work (i.e. those working primarily in outdoor/public spaces). In operation since 1987, WISH is the only late night drop-in space for female FSWs that is open 7 nights a week (5:00 PM to 11:00 PM daily until 2013, when the hours were extended to 7:00 AM). It serves approximately 200 women per night and provides low-threshold services, such as hot meals, showers, hygiene items, clothing, harm reduction and safety supplies (e.g. bad date sheets, condoms, and clean syringes), and referrals to social and health support services. In addition to these core services, WISH offers ongoing peer/FSW education and support programs, and clinics run by outreach nurses and nurse practitioners provide onsite basic primary care and referrals.

The aim of the present study was to longitudinally examine the uptake of WISH services over a 3-year observation period, and to longitudinally evaluate the relationship between WISH utilization and SRH service access for FSWs.

\section{Materials and methods}

For the present longitudinal analysis, data were drawn from an open prospective cohort, An Evaluation of Sex Workers' Health Access (AESHA), which initiated recruitment in January 2010. AESHA is a community-based study that was initiated in 2005 and has been developed and guided through longstanding collaborations with sex work, women's, and health services agencies and formative research [18]. The cohort includes female individuals (including transgender women) in Vancouver, BC, Canada, who are aged 14 years or older and have exchanged sex for money or resources within the last 30 days. All participants provide written informed consent. For the present study, data obtained between January 2010 and February 2013 were analyzed. The AESHA study holds ethical approval through Providence Health Care/University of British Columbia Research Ethics Board and is conducted according to the principles of the Declaration of Helsinki and Canadian TriCouncil Policy guidelines.

Because of the difficulties of recruiting stigmatized and isolated populations such as FSWs, as previously described [18], FSWs are recruited for the AESHA study using time-location sampling. A team of interviewers and outreach workers (including staff with sex work experience) conduct weekly daytime and late-night outreach visits to sex work "strolls" (public sex work locations [streets and alleys]), indoor sex work venues (hotels, massage parlors, micro-brothels), and other in-and-out call locations (e.g. private homes and bars) and online/self-advertising spaces across Metro Vancouver. A list of street and indoor sex work venues is identified through community mapping conducted with current/former FSWs [18] and regularly updated. 
After enrollment, participants are followed up every 6 months. Follow-up visits take place at one of two study office locations, or at a safe and confidential location as identified by the participant. Participants receive CAN\$40 at every semi-annual visit for their time, expertise, and travel.

At baseline and follow-up visits, a trained interviewer administers a main questionnaire to participants, which obtains information about individual characteristics, work environment, partner/dyad sexual risks, and protective factors (e.g. condom use and access to harm reduction). Additionally, a project nurse administers a counseling questionnaire before performing HIV/STI testing. The counseling questionnaire obtains information about physical, sexual, and mental health. Patterns of healthcare and social support use are also recorded. SRH services assessed include contraceptives, obstetric consultations, cervical smears, and HIV and STI testing, all of which can be accessed through community clinics, hospital, or outreach services.

Biolytical INSTI (Biolytical Laboratories Inc, Richmond, BC, Canada) rapid tests are used for HIV screening; reactive tests are confirmed by western blot at the British Columbia Centre for Disease Control (Vancouver, BC, Canada). Urine samples are tested for gonorrhea and chlamydia. Blood samples are tested for syphilis via rapid plasma reagin, and a Treponema pallidum hemagglutination assay is conducted for positive samples. All patients receive post-test counseling and treatment as needed, as well as referrals to services (e.g. drop-in services [including WISH] and harm reduction services).

The present analysis was restricted to FSWs who did not primarily work in more established venues (e.g. massage parlors), because WISH services are not tailored toward FSWs in more organized segments of the sex industry. The dependent variable for the study was a timeupdated measure of use of WISH, based on a "yes" response at baseline and semi-annual follow-up visits to a serial measure of having accessed any services or resources at WISH in the previous 6 months.

Descriptive statistics (i.e. frequencies, proportions, medians, and interquartile ranges [IQRs]) for the baseline characteristics were calculated and stratified according to WISH use at baseline. Baseline characteristics were assessed using Pearson's $\chi^{2}$ test for categorical variables and the Mann-Whitney test for continuous variables.

For longitudinal analyses, sociodemographic variables were considered as fixed variables. All other variables were time-varying, on the basis of repeated measures collected at semiannual visits over the study period. Correlates of using WISH were examined using bivariable and multivariable generalized estimating equations (GEEs) with a logit link function for the dichotomous outcome. To adjust the standard error and account for correlations arising from repeated measurements on the same participant over time, an exchangeable correlation matrix was used. Variables chosen a priori and significant at $P<0.05$ in bivariable analysis were considered for inclusion in the multivariable model. Backward model selection and quasi-likelihood under the independence model criterion value were used to build the final multivariable model, as has been done previously [18]. Two-sided $P$ values, and unadjusted and adjusted odds ratios with 95\% confidence intervals 
are reported. Statistical analyses were performed using SAS version 9.3 (SAS Institute, Cary, NC, USA).

\section{Results}

Of 547 FSWs included in the present analysis, 269 (49.2\%) had used WISH in the 6 months before baseline interview. At baseline, FSWs who had accessed WISH services in the previous 6 months were older and more likely to be of Aboriginal ancestry than were those who had not used WISH $(P<0.05)$ (Table 1$)$. Furthermore, participants who had recently used WISH were more likely to report using injection and non-injection drugs ( $P \unlhd 0.001$ ) (Table 1). More than three-quarters of the participants had ever been pregnant (Table 1).

Of the 547 participants, 425 (77.7\%) had returned for at least one follow-up visit, with medians of 3 visits (IQR 2-4) and 23.85 months (IQR 13.08-29.93) of follow-up. Additionally, 330 (60.3\%) visited WISH at some point during the 3-year study period. The services most frequently accessed at WISH were food provision, make-up, clothing, and primary nursing care (Table 2).

In unadjusted GEE analysis, HIV seropositivity was significantly associated with visiting WISH in the 3-year study period (Table 3). Furthermore, FSWs who visited WISH were more likely to be of Aboriginal ancestry, have been born in Canada, be homeless, use injection and non-injection drugs, exchange sex for drugs, and work primarily in outdoor/ public spaces (Table 3). They were also more likely to report physical/sexual violence by clients, accessing SRH services, and hospitalization for a health issue (Table 3). In the multivariable GEE model, variables that retained an independent correlation with accessing WISH were age, Aboriginal ancestry, injection drug use, exchange of sex for drugs, and accessing SRH services (Table 3).

\section{Discussion}

The present study has shown high uptake of a women-only and sex-work-specific drop-in space for street-involved FSWs and demonstrates that this model can effectively link marginalized women with SRH services. Despite ample evidence suggesting high SRH morbidity and barriers to access among FSWs, few investigations have examined the effect of women-only and sex-work-specific services on access to SRH services. The present data support evidence from LMIC settings that women-centered and sex-work-specific community strategies and low-threshold support services (e.g. drop-in centers and outreach) can successfully link highly stigmatized populations with healthcare services [6].

In view of the barriers FSWs encounter when attempting to access conventional health services, women-centered and sex-work-specific drop-in spaces can provide safe, nonjudgmental and "enabling environments" in which health and social services can be accessed, and link women with mainstream care [19]. These successful FSW-led and sexwork-specific programming models are based on community empowerment, which is defined as a "collective process through which the structural constraints to health, human rights, and well-being are addressed by sex workers to create social and behavioral changes, and access to health services" [6]. The Sonagachi Project, which is based in Calcutta, India, 
has demonstrated that collectivization, empowerment, and education all play crucial parts in the promotion of HIV/STI prevention and care [20]. A key pillar of the Sonagachi Project's success was the role of FSWs as peer educators to disseminate relevant information about sexual and reproductive health.

Alongside evidence from LMIC settings, the role of occupational health and safety clinics has been considered in high-income settings. The St. James Infirmary in San Francisco, CA, USA, is a program with substantial FSW leadership that provides nonjudgmental sex-workspecific occupational health care and social services, including SRH care, peer counseling, needle exchange, and primary care [21]. In Vancouver, several community- and hospitalbased models for pregnant women and women who have recently delivered (e.g. Sheway and Fir Square) have made important progress for individuals who use drugs; however, broader SRH services, alongside occupational health, for FSWs remain largely absent.

Notably, the present results suggest that WISH is successfully reaching a large proportion of the most marginalized street-involved FSWs, including those who are HIV seropositive, of Aboriginal ancestry, and use injection drugs. Within overlapping street-based drug and sex work scenes, this marginalized subpopulation of FSWs faces a disproportionate burden of barriers to conventional healthcare services and poor SRH outcomes $[8,18]$. For example, the present study found that WISH users are less likely to use modern methods of contraception; their greater health and social vulnerabilities could relate to lower awareness and access to modern contraception. Taken together, the results suggest that low-threshold service delivery models, such as drop-in spaces and outreach services, remain crucial strategies for linking marginalized populations with health care, including FSWs and people who use drugs [20,22,23]. Health services should also focus on eliminating barriers to sexwork-specific programs for younger FSWs, who are less likely to access services, as evidenced by this study and previous findings [24]. Furthermore, decriminalization of sex work and recognition of sex work as a legitimate occupation are important steps toward reducing barriers of stigma and discrimination to critical health and social services [25].

Several potential limitations should be considered when interpreting the results of the present study. First, achieving a representative sample is challenging for marginalized and isolated populations such as FSWs. However, the outreach team includes both FSWs and community members with extensive experience in working with FSWs. Time-location sampling and community mapping were used to ensure broad representation of FSWs from all street and off-street venues. Second, although the present study draws on prospective data and uses GEEs and a working correlation matrix to account for repeated measures by the same respondent, it is not possible to determine causality. However, the data support a clear independent correlation between the use of the WISH and SRH services. Finally, as with other observational research, responses may be subject to social desirability bias; however, there is no reason to assume that there would be differences in reporting between FSWs accessing and not accessing WISH.

In summary, the present results indicate a high uptake of a women-only and sex-workspecific drop-in center and an independent longitudinal correlation with access to SRH care among street-involved FSWs. However, it is important to note that only $60 \%$ of FSWs 
accessed WISH in the 3-year observation period, and barriers to access remain for others, including geography and displacement due to policing of street-based sex work [11]. These findings suggest that policy and program support are needed to scale up low-threshold and sex-work-specific models for integrated SHR, alongside community and social support models. Successful models of FSW-led and sex-work-specific services from LMIC settings and occupational health and safety clinics in the USA hold promise for models that could be adapted and assessed in the Canadian context.

\section{Acknowledgments}

This research was supported by operating grants from the US National Institutes of Health (R01DA028648) and Canadian Institutes of Health Research (HHP-98835). S.G. and K.S. are supported by the Canadian Institutes of Health Research and Michael Smith Foundation for Health Research/Women's Health Research Institute.

\section{References}

1. United Nations Population Fund. [Accessed June 1, 2014] Human rights: supporting the constellation of reproductive rights. http://www.unfpa.org/rights/rights.htm

2. Sarna A, Friedland BA, Srikrishnan AK, Katzen LL, Tun W, Abbott SA, et al. Sexually transmitted infections and reproductive health morbidity in a cohort of female sex workers screened for a microbicide feasibility study in Nellore, India. Glob J Health Sci. 2013; 5(3):139-49. [PubMed: 23618483]

3. McDougal L, Strathdee SA, Rangel G, Martinez G, Vera A, Sirotin N, et al. Adverse pregnancy outcomes and sexual violence among female sex workers who inject drugs on the United StatesMexico border. Violence Vict. 2013; 28(3):496-512. [PubMed: 23862312]

4. Perla ME, Ghee AE, Sánchez S, McClelland RS, Fitzpatrick AL, Suárez-Ognio L, et al. Genital tract infections, bacterial vaginosis, HIV, and reproductive health issues among Lima-based clandestine female sex workers. Infect Dis Obstet Gynecol. 2012; 2012:739624. [PubMed: 22811592]

5. Decker MR, Yam EA, Wirtz AL, Baral SD, Peryshkina A, Mogilnyi V, et al. Induced abortion, contraceptive use, and dual protection among female sex workers in Moscow, Russia. Int J Gynecol Obstet. 2013; 120(1):27-30.

6. World Health Organization. [Accessed March 1, 2014] Prevention and treatment of HIV and other sexually transmitted infections for sex workers in low- and middle-income countries: recommendations for a public health approach. http://www.who.int/hiv/pub/guidelines/sex_worker/ en/. Published December 2012

7. Porras C, Sabidó M, Fernández-Dávila P, Fernández VH, Batres A, Casabona J. Reproductive health and healthcare among sex workers in Escuintla, Guatemala. Cult Health Sex. 2008; 10(5): 529-38. [PubMed: 18568874]

8. Duff P, Shoveller J, Zhang R, Alexson D, Montaner JS, Shannon K. High lifetime pregnancy and low contraceptive usage among sex workers who use drugs-an unmet reproductive need. BMC Pregnancy \& Childbirth. 2011; 11:61. [PubMed: 21851622]

9. Krüsi A, Chettiar J, Ridgway A, Abbott J, Strathdee SA, Shannon K. Negotiating safety and sexual risk reduction with clients in unsanctioned safer indoor sex work environments: a qualitative study. Am J Public Health. 2012; 102(6):1154-9. [PubMed: 22571708]

10. Phrasisombath K, Thomsen S, Sychareun V, Faxelid E. Care seeking behaviour and barriers to accessing services for sexually transmitted infections among female sex workers in Laos: a crosssectional study. BMC Health Serv Res. 2012; 12:37. [PubMed: 22333560]

11. Duff P, Shoveller J, Chettiar J, Feng C, Nicoletti R, Shannon K. Sex work and motherhood: social and structural barriers to health and social services for pregnant and parenting street- and off-street sex workers. Health Care Women Int. In Press.

12. Chen XS, Yin YP, Liu GG, Wei WH, Wang HC, Yu YL, et al. Outreach syphilis testing services by different health providers to female sex workers in southern China. PLoS One. 2013; 23;8(4):e60626. 
13. Moore E, Han J, Serio-Chapman C, Mobley C, Watson C, Terplan M. Contraception and clean needles: feasibility of combining mobile reproductive health and needle exchange services for female exotic dancers. Am J Public Health. 2012; 102(10):1833-6. [PubMed: 22897527]

14. Luchters S, Chersich MF, Rinyiru A, Barasa MS, King'ola N, Mandaliya K, et al. Impact of five years of peer-mediated interventions on sexual behavior and sexually transmitted infections among female sex workers in Mombasa, Kenya. BMC Public Health. 2008; 8:143. [PubMed: 18445258]

15. Parimi P, Mishra RM, Tucker S, Saggurti N. Mobilising community collectivisation among female sex workers to promote STI service utilization from the government healthcare system in Andhra Pradesh, India. J Epidemiol Community Health. 2012; 66(suppl 2):ii62-8. [PubMed: 22493478]

16. Chacham AS, Diniz SG, Maia MB, Galati AF, Mirim LA. Sexual and reproductive health needs of sex workers: two feminist projects in Brazil. Reprod Health Matters. 2007; 15(29):108-18. [PubMed: 17512382]

17. Lafort Y, Geelhoed D, Cumba L, Lázaro Cd, Delva W, Luchters S, et al. Reproductive health services for populations at high risk of HIV: performance of a night clinic in Tete province, Mozambique. BMC Health Serv Res. 2010; 10:144. [PubMed: 20507644]

18. Shannon K, Bright V, Allinott S, Alexson D, Gibson K, Tyndall MW, et al. Community-based HIV prevention research among substance-using women in survival sex work: the Maka Project Partnership. Harm Reduct J. 2007; 4:20. [PubMed: 18067670]

19. Deering KN, Shannon K, Sinclair H, Parsad D, Gilbert E, Tyndall MW. Piloting a peer-driven intervention model to increase access and adherence to antiretroviral therapy and HIV care among street-entrenched HIV-positive women in Vancouver. AIDS Patient Care STDs. 2009; 23(8):6039. [PubMed: 19591602]

20. Gangopadhyay DN, Chanda M, Sarkar K, Niyogi SK, Chakraborty S, Saha MK, et al. Evaluation of sexually transmitted diseases/human immunodeficiency virus intervention programs for sex workers in Calcutta, India. Sex Transm Dis. 2005; 32(11):680-4. [PubMed: 16254542]

21. Cohan D, Lutnick A, Davidson P, Cloniger C, Herlyn A, Breyer J, et al. Sex worker health: San Francisco style. Sex Transm Infect. 2006; 82(5):418-22. [PubMed: 16854996]

22. Coyle SL, Needle RH, Normand J. Outreach-based HIV prevention for injecting drug users: a review of published outcome data. Public Health Rep. 1998; 113(Suppl 1):19-30. [PubMed: 9722807]

23. Deering KN, Kerr T, Tyndall MW, Montaner JS, Gibson K, Irons L, et al. A peer-led mobile outreach program and increased utilization of detoxification and residential drug treatment among female sex workers who use drugs in a Canadian setting. Drug Alcohol Depend. 2011; 113(1):4654. [PubMed: 20727683]

24. Goldenberg SM, Chettiar J, Simo A, Silverman JG, Strathdee SA, Montaner JS, et al. Early sex work initiation independently elevates odds of HIV infection and police arrest among adult sex workers in a Canadian setting. J Acquir Immune Defic Syndr. 2014; 65(1):122-8. [PubMed: 23982660]

25. Lazarus L, Deering KN, Nabess R, Gibson K, Tyndall MW, Shannon K. Occupational stigma as a primary barrier to health care for street-based sex workers in Canada. Cult Health Sex. 2012; 14(2):139-50. [PubMed: 22084992] 


\section{Synopsis}

High uptake of sex-work-specific, low-threshold drop-in services can effectively link marginalized women with sexual and reproductive health care. 


\section{Table 1}

Baseline characteristics. ${ }^{a}$

\begin{tabular}{|c|c|c|c|c|}
\hline \multirow[t]{2}{*}{ Characteristic } & \multirow[t]{2}{*}{ All sex workers $(n=547)$} & \multicolumn{2}{|c|}{ Used WISH within previous 6 months } & \multirow[t]{2}{*}{$P$ value } \\
\hline & & Yes $(n=269)$ & No $(n=278)$ & \\
\hline \multicolumn{5}{|l|}{ Individual/sociodemographic } \\
\hline Age, $y$ & $33(27-42)$ & $35(29-43)$ & $33(26-40)$ & 0.002 \\
\hline Aboriginal ancestry $b$ & $247(45.2)$ & $151(56.1)$ & $96(34.5)$ & $<0.001$ \\
\hline Born in Canada & $488(89.2)$ & $260(96.7)$ & $228(82.0)$ & $<0.001$ \\
\hline LGBTQ & $170(31.1)$ & $81(30.1)$ & $89(32.0)$ & 0.631 \\
\hline Completed high school or further education & $233(42.6)$ & $100(37.2)$ & $133(47.8)$ & 0.012 \\
\hline Homeless $c$ & $213(38.9)$ & $111(41.3)$ & $102(36.7)$ & 0.273 \\
\hline Injection drug use ${ }^{c}$ & $277(50.6)$ & $156(58.0)$ & $121(43.5)$ & 0.001 \\
\hline Non-injection drug use ${ }^{c}$ & $470(85.9)$ & $248(92.2)$ & $222(79.9)$ & $<0.001$ \\
\hline \multicolumn{5}{|l|}{ Health status } \\
\hline HIV seropositivity & $78(14.3)$ & $50(18.6)$ & $28(10.1)$ & 0.004 \\
\hline STI seropositivity & $71(13.0)$ & $36(13.4)$ & $35(12.6)$ & 0.783 \\
\hline Diagnosis of mental health issue (ever) & $314(57.4)$ & $162(60.2)$ & $152(54.7)$ & 0.190 \\
\hline \multicolumn{5}{|l|}{ Partner/dyad sexual risks ${ }^{c}$} \\
\hline Inconsistent condom use by male client & $121(22.1)$ & $66(24.5)$ & $55(19.8)$ & 0.181 \\
\hline Exchange of sex for drugs & $208(38.0)$ & $110(40.9)$ & $98(35.3)$ & 0.174 \\
\hline Physical/sexual violence by client & $152(27.8)$ & 77 (28.6) & $75(27.0)$ & 0.667 \\
\hline \multicolumn{5}{|l|}{ Work environment ${ }^{c}$} \\
\hline Street/public space & $307(56.1)$ & $158(58.7)$ & $149(53.6)$ & Reference \\
\hline Informal indoor venues (e.g. bars, hotels, saunas) & $178(32.5)$ & $92(34.2)$ & $86(30.9)$ & 0.963 \\
\hline Formal indoor venues (e.g. brothels) & $62(11.3)$ & $19(7.1)$ & $43(15.5)$ & 0.003 \\
\hline \multicolumn{5}{|l|}{ Sexual and reproductive health outcomes } \\
\hline Use of barrier contraceptives $c$ & $359(65.6)$ & $175(65.1)$ & $184(66.2)$ & 0.781 \\
\hline Use of modern contraceptives $c, d$ & $103(18.8)$ & $41(15.2)$ & $62(22.3)$ & 0.035 \\
\hline Use of permanent contraceptives $c$ & $36(6.6)$ & $21(7.8)$ & $15(5.4)$ & 0.256 \\
\hline Pregnancy (ever) & $420(76.8)$ & $208(77.3)$ & $212(76.3)$ & 0.768 \\
\hline Number of unintended pregnancies & $2(0-3)$ & $2(0-3)$ & $1.5(0-4)$ & 0.563 \\
\hline \multicolumn{5}{|l|}{ Access to care ${ }^{c}$} \\
\hline Sexual and reproductive health services & $198(36.2)$ & $124(46.1)$ & 74 (26.6) & $<0.001$ \\
\hline Sought care for a health issue & $364(66.5)$ & $189(70.3)$ & $175(62.9)$ & 0.070 \\
\hline Hospitalization (overnight or longer) & $119(21.8)$ & $75(27.9)$ & $44(15.8)$ & 0.001 \\
\hline Experienced barriers to primary health care & $362(66.2)$ & $172(63.9)$ & $190(68.3)$ & 0.276 \\
\hline
\end{tabular}

Abbreviations: WISH, Women's Information Safe Haven Drop-In Centre; LGBT, lesbian, gay, bisexual, transgender, or other sexual minority (e.g. transsexual, two-spirited, or other); STI, sexually transmitted infection.

${ }^{a}$ Values are given as median (interquartile range) or number (percentage), unless indicated otherwise.

${ }^{b}$ First Nations, Métis, Inuit, and non-status First Nations. 
${ }^{c}$ All variables refer to activities in last 6 months of baseline interview.

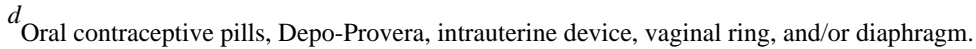




\section{Table 2}

Services used at WISH by female sex workers during the study period $(n=330)$.

\begin{tabular}{lc}
\hline Types of services & No. $(\%)$ \\
\hline Medical services & \\
Primary nursing care & $93(28.2)$ \\
Referral to a healthcare professional & $43(13.0)$ \\
Non-medical services & \\
Food & $313(94.8)$ \\
Make-up & $205(62.1)$ \\
Clothing & $97(29.4)$ \\
Shower & $16(4.8)$ \\
Counselor service & $36(10.9)$ \\
Peer-support/education program & $23(7.0)$ \\
\hline
\end{tabular}

Abbreviation: WISH, Women's Information Safe Haven Drop-In Centre. 
Table 3

Bivariable and multivariable generalized estimating equations analyses for correlates of using WISH.

\begin{tabular}{|c|c|c|c|c|}
\hline \multirow[t]{2}{*}{ Characteristic } & \multicolumn{2}{|c|}{ Unadjusted } & \multicolumn{2}{|c|}{ Adjusted } \\
\hline & OR $(95 \% \mathrm{CI})$ & $P$ value & OR $(95 \% \mathrm{CI})$ & $P$ value \\
\hline \multicolumn{5}{|l|}{ Individual/sociodemographic } \\
\hline $\mathrm{Age}^{a}$ & $1.03(1.02-1.05)$ & $<0.001$ & $1.04(1.03-1.06)$ & $<0.001$ \\
\hline Aboriginal ancestry & $1.94(1.46-2.56)$ & $<0.001$ & $2.18(1.61-2.95)$ & $<0.001$ \\
\hline Born in Canada & $4.61(2.51-8.47)$ & $<0.001$ & - & - \\
\hline High school or postsecondary education & $0.65(0.49-0.87)$ & 0.003 & - & - \\
\hline Homeless $b$ & $1.32(1.08-1.62)$ & 0.007 & - & - \\
\hline Injection drug use $b$ & $1.88(1.46-2.41)$ & $<0.001$ & $1.67(1.29-2.17)$ & $<0.001$ \\
\hline Non-injection drug use ${ }^{b}$ & $1.74(1.24-2.44)$ & 0.001 & - & - \\
\hline \multicolumn{5}{|l|}{ Health status } \\
\hline HIV seropositivity & $1.66(1.17-2.35)$ & 0.004 & - & - \\
\hline STI seropositivity & $1.03(0.76-1.38)$ & 0.866 & - & - \\
\hline \multicolumn{5}{|l|}{ Partner/dyad sexual risks $b$} \\
\hline Exchange of sex for drugs & $1.65(1.37-1.98)$ & $<0.001$ & $1.40(1.15-1.71)$ & 0.001 \\
\hline Physical/sexual violence by client & $1.35(1.08-1.68)$ & 0.007 & - & - \\
\hline \multicolumn{5}{|l|}{ Work environment $b$} \\
\hline Street/public space & Reference & - & Reference & - \\
\hline Informal indoor venues (e.g. bars, hotels, saunas) & $0.82(0.68-0.98)$ & 0.031 & $0.83(0.68-1.00)$ & 0.051 \\
\hline Formal indoor venues (e.g. brothels) & $0.57(0.38-0.86)$ & 0.008 & $0.67(0.45-1.01)$ & 0.054 \\
\hline \multicolumn{5}{|l|}{ Access to care ${ }^{b}$} \\
\hline Sexual and reproductive health services & $1.97(1.62-2.40)$ & $<0.001$ & $1.65(1.35-2.02)$ & $<0.001$ \\
\hline Sought care for a health issue & $1.16(0.97-1.40)$ & 0.109 & - & - \\
\hline Hospitalization (overnight or longer) & $1.20(0.98-1.49)$ & 0.083 & - & - \\
\hline Experienced barriers to primary health care & $1.28(1.09-1.52)$ & 0.003 & - & - \\
\hline
\end{tabular}

Abbreviations: WISH, Women's Information Safe Haven Drop-In Centre; OR, odds ratio; CI, confidence interval; STI, sexually transmitted infection.

${ }^{a}$ Per increase of 1 year.

${ }^{b}$ All variables are serial, time-updated at each study visit, using the last 6 months as a reference point. 